\title{
PAIR UP for primary care excellence: perspectives from a primary healthcare provider in Singapore
}

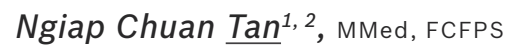

\begin{abstract}
Singapore is facing an increasing noncommunicable disease burden due to its ageing population. Singapore's primary healthcare services, provided by both polyclinic physicians and private general practitioners, are available to the public at differential fees for service. The resultant disproportionate patient loads lead to dissatisfaction for both healthcare providers and consumers. This article describes the 'PAIR UP' approach as a potential endeavour to facilitate primary care physicians (PCPs) in public and private sectors to collaborate to deliver enhanced primary care in Singapore. PAIR UP is an acronym referring to Policy, Academic development, Integration of healthcare information system, Research in primary care, Utility and safety evaluation, and Practice transformation. The current healthcare landscape is favourable to test out this multipronged approach. PCPs in both sectors can ride on it and work together synergistically to provide quality primary care in Singapore.
\end{abstract}

Keywords: care integration, policy, practice transformation, primary care

\section{INTRODUCTION}

Faced with a rapidly ageing population, 15\% of Singapore's population will be 65 years and older by 2020. ${ }^{(1)}$ With this, there will be a natural parallel rise in the prevalence of chronic diseases among the elderly. As primary care centres are the first point of contact for the majority of patients, most elderly patients will seek treatment for these diseases in the primary care setting. The Primary Care Survey in 2010 revealed that compared to $86 \%$ of primary care physicians (PCPs) working in the private general practitioner (GP) sector, $14 \%$ of all PCPs work in polyclinics, where $19 \%$ of all patient attendances were reported to take place. ${ }^{(2)}$ This smaller proportion of doctors working in polyclinics was found to manage $45 \%$ of all patient attendances for chronic disease management. ${ }^{(2)}$ Based on our current primary care model, this significant disparity can be attributed to the availability of subsidised treatment and a wider range of ambulatory services in polyclinics, which leads to overcrowding, prolonging the waiting time for patients.

To avoid compromising the quality of primary care in Singapore, it is critical to 'PAIR UP' public and private PCPs. This article describes a collaborative approach in which PCPs work together synergistically to deliver quality primary care to Singapore's population.

\section{PAIR UP APPROACH}

PAIR UP - Policy, Academic development, Integration of healthcare information system, Research in primary care, Utility and safety evaluation, and Practice transformation - is a sixpronged strategy aimed at achieving primary care excellence by enhancing the synergy between public and private PCPs. This approach creates opportunities for healthcare providers to reach out to the local population effectively amidst Singapore's changing healthcare landscape. This will also help to level the chronic disease burden in the community and ensure that access to timely, affordable and quality primary care continues for the ageing population.

\section{Policy}

Singapore's primary healthcare system largely operates under a fee-for-service, walk-in model, in which patients are able to select their preferred PCP at either public polyclinics or private GP clinics. Although the cost of treatment is subsided at polyclinics, patients still have to pay a portion of their consultation fees. In contrast, patients attending private GP clinics do not receive any subsidies and have to bear the full cost of treatment. However, patients can choose to use the funds in their Medisave account to partially offset the bill for selected disease treatment and services at both GP clinics and polyclinics. A key component of the ' $3 \mathrm{M}$ ' healthcare financing structure, Medisave comprises mandatory healthcare savings that are contributed to by patients, and their employers or relatives. The other two components of the $3 \mathrm{M}$ structure are Medishield, a catastrophic medical insurance scheme, and Medifund, a means-tested financial assistance scheme. ${ }^{(3)}$ Several landmark shifts in the Medisave scheme had set the pace for the significant changes in primary care that were effected in the last two years. ${ }^{(4)}$

With the initiation of the Chronic Disease Management Programme (CDMP) in recent years, patients are able to use the funds in their Medisave accounts to pay for outpatient treatment of selected chronic diseases, ranging from asthma to arthritis, in both public and private primary care clinics registered with the Ministry of Health, Singapore. ${ }^{(4)}$ The cost

${ }^{1}$ Department of Research, SingHealth Polyclinics, ${ }^{2}$ Duke-NUS Graduate Medical School, Singapore

Correspondence: Dr Tan Ngiap Chuan, Director, Department of Research, SingHealth Polyclinics Head Office, 167 Jalan Bukit Merah, Connection One, Tower 5, \#15-10, Singapore 150167. tan.ngiap.chuan@singhealth.com.sg 
of medical treatment can be partially offset using the funds in patients' Medisave accounts. ${ }^{(4)}$ This allows private GPs to help manage the increasing number of patients with chronic diseases in the community, who may be constrained by the opening hours of polyclinics. The administrative charges accompanying the use of Medisave funds for these patients have also been removed, reducing another barrier in the utilisation of Medisave funds. ${ }^{(4)}$

Another remarkable development in the local healthcare landscape is the launch of the Community Health Assist Scheme (CHAS), which extends healthcare cost subsidies to patients visiting private GPs. ${ }^{(5)}$ Under CHAS, patients are meanstested based on their per capita monthly household income in order to determine whether they are eligible for subsidies for consultation and treatment at CHAS-registered private GP clinics. A portable healthcare subsidy operated by the Agency for Integrated Care (AIC), CHAS covers the following: (a) medications commonly used to treat various prevalent chronic diseases; (b) preventive measures such as influenza and pneumococcal vaccinations; and (c) subsidised referral to specialists in public hospitals by private GPs included under the scheme. Revision of the age limit and monthly household income ceiling for eligible patients who qualify for inclusion under CHAS, and extension of the subsidy to their children without further means testing are some recent enhancements aimed at enabling a wider pool of patients in the community to be treated by private GPs in their neighbourhood. ${ }^{(5)}$

Our healthcare infrastructure is compartmentalised into six regional healthcare systems (RHS), comprising public primary, secondary, tertiary and transitional healthcare institutions. ${ }^{(6)}$ Each RHS sets out to establish a network of private GP clinics in their respective localities to facilitate the discharge of stable patients from hospital care to primary care. ${ }^{(6)}$ With CHAS in place, private GPs and their polyclinic counterparts assume an increasingly important role in providing continuity of care to ensure seamless transition of patients from hospitals to the community. To allow for holistic patient care, each RHS has developed community healthcare resources to enable private GPs (within their respective RHS network) to have more responsive access to facilities for the management of chronic or complex diseases such as diabetes mellitus. ${ }^{(6)}$ Private GPs also have the option of referring these patients to community health centres (CHCs) for diabetes-related eye and foot screenings, thus leveraging well-trained nurse counsellors at these $\mathrm{CHCs}$ to better educate and support patients with regard to the adoption of lifestyle and behavioural changes. ${ }^{(7,8)}$

Another new primary care establishment is the Family Medicine Clinic (FMC), which aims to provide an environment conducive for the delivery of comprehensive care to patients with chronic diseases. ${ }^{(9)}$ Much like a mini-polyclinic, FMCs are well equipped with facilities and incorporated with a patient information system integrated with the respective RHS, and are designed to be operated by a smaller number of private GPs.
FMCs are also staffed with healthcare professionals, such as nurse counsellors, to provide core services such as diabetic eye and foot screening. With a focus on chronic care and seamless integration into the RHS, the FMC concept is a plausible business model for private GPs, but its viability needs to be time-tested.

\section{Academic development}

The Register of Family Physicians (FPR) was set up in July 2011 to recognise physicians from public and private primary care clinics with relevant training and qualifications for continuous professional development. ${ }^{(10)}$ FPR candidates have to be accredited by the Family Physicians Accreditation Board and the Singapore Medical Council. ${ }^{(10)}$ With the restructuring of primary care in Singapore, FPR provides a platform through which standards for the practice of family medicine can be set. The FPR aims to encourage PCPs to take on postgraduate training in family medicine and be equipped with the relevant skills to manage the increasingly complex complications of an ageing population.

In addition, the family medicine training programme of junior doctors who aspire to become family physicians has undergone a significant revamp and is replaced by the Residency programme accredited under the Accreditation Council for Graduate Medical Education (ACGME) system. ${ }^{(11)}$ Its structured three-year programme is based on four key components: (a) a framework of regulation; (b) a well-defined curriculum; (c) designated faculty staff and system of regular formative assessment; and (d) summative assessments for residents. The curriculum encompasses training in six core competencies medical knowledge, patient care, professionalism, interpersonal skills and communication, practice-based learning, and systembased practice. Its comprehensive training activities include clinical training, lectures, tutorials, workshops, and project involvement such as quality improvement and research projects. Residents have to undertake core and elective rotations, and clinical attachments in various speciality disciplines in order to attain the comprehensive and challenging skill sets needed to be competent family physicians. Residents who complete this programme will proceed to pursue a fruitful career in either public or private primary care clinics, advance the discipline of family medicine, and handle the wide clinical scope that comes with family practice.

Beyond the fundamentals of general practice, family physicians from both the public and private sectors can continue to pursue professional development in advanced family medicine training programmes. The Family Medicine Fellowship Programme, developed by the College of Family Physicians Singapore (CFPS) aims to groom family physicians into leaders in family medicine. ${ }^{(12)}$ In line with the family medicine philosophy, fellows are expected to tackle the evolving demands of family medicine, manage the challenges of transitional care, optimise and coordinate the use of community resources, and establish 
themselves as role models to junior doctors and aspirant family physicians.

\section{Integration of healthcare information system}

Physicians from polyclinics and only selected GPs can gain access to patients' health records via the National Electronic Health Records (NEHR). ${ }^{(13)}$ However, patients may consult both polyclinic doctors and private GPs due to the different operation modes of public and private primary care clinics. To potentially circumvent this, access to NEHR can be granted to all private GPs in the near future, with the consent of their respective patients. With this, both public and private PCPs can leverage an integrated NEHR to have a more complete understanding of the health status of their patients, which is essential for the provision of holistic care. In this way, safety and healthcare resources are optimised, as a common patient health record will avoid duplicate investigations and treatment.

A parallel development to this is the current construction of MOH Holdings' National Patient Health Management portal, known as Healthy.SG. ${ }^{(14)}$ With the aim of motivating patients to take responsibility for their own well-being, this portal will be a web-based platform for patients to capture their personal health data. This device-agnostic portal facilitates personal health monitoring, as clinical data can be transmitted directly to the portal via various devices such as mobile phones and computers. Personal health awareness can be enhanced by patients' own reflection of clinical data such as home blood pressure and glucose monitoring readings, calorie consumption from diet, and calorie expenditure from exercise. This multichannel communication portal also allows patients to transmit such data not only to family members or friends for social support and mutual motivation, but also to their designated family physicians and healthcare professionals. With this platform, public and private PCPs can use such timely and actionable health information to build trust and long-term partnerships with their patients.

\section{Research in primary care}

Research in primary care has been shown to improve global health, which translates into improved quality of primary care and often a reduction in national healthcare expenditure. ${ }^{(15)}$ Thus, it is important that PCPs have a basic understanding of how research is performed. Research-related training will enable understanding of the key processes involved in each step of the research cycle - from the formulation of the research question and deliberation on the use of the appropriate research design method to answer the question, to data collection and computation, and analysis and interpretation of the results. Research is incorporated as a training module in the CFPS Family Medicine Fellowship programme and $\mathrm{MOH}$ Residency programmes. ${ }^{(11,12)}$ Fellows are expected to embark on a research project as part of their curriculum, providing them with the opportunity for hands-on experience in research. Residents may collaborate with senior family physicians as part of a research team to gain research exposure.

Regardless of their practice setting, PCPs are encouraged to initiate research that is specific and applicable to the primary healthcare system. ${ }^{(12)}$ However, what is often published are reports using data from secondary and tertiary institutions, in very controlled environments, which may not be easily translated into enhancements in primary care practices. ${ }^{(16,17)}$ Giveon et al showed that Israeli family medicine residents coached by proresearch mentors were more actively involved in research after qualifying as family physicians. ${ }^{(18)}$ Thus, the appointment of mentors who are experienced family medicine researchers, as done by CFPS, would enhance the support of family medicine fellows, facilitating the transformation of relevant and significant clinical issues into answerable research questions, ${ }^{(12)}$ resulting in fellows who are trained to use appropriate research designs to determine the answers. Ultimately, the main aim of such an approach is to train PCPs to use research to bridge the gaps in their knowledge, so as to improve patient care and enhance primary care practices.

Nonetheless, participation in research will be a challenge for the majority of practising PCPs, due to time and resource constraints, and a lack of mentorship. ${ }^{(19,20)}$ CFPS has, however, recently launched a research network aimed at allowing PCPs to immerse in practice-based research, ${ }^{(21)}$ increasing research bandwidth by expanding the number of research sites and the capacity of data collection. Also known as INSPIRE (i.e. Integrated Network of Singapore Primary care physicians in Research Endeavour), this network can potentially advance family medicine research in Singapore through multilateral collaborations and leverage the economy of scale of a practicebased network. Start-up or seed funding from CFPS and other sources for private GPs will also further increase this capability. A public primary care institution has also set up a research consultation clinic to provide advice to aspiring family medicine researchers with respect to the steps of the research cycle. ${ }^{(22)}$ Private GPs can also tap on this resource, which is extended to them free of charge. ${ }^{(22)}$

Altruism aside, longer-term sustainability depends on the fundamental structure upon which the national funding of primary care research is built. The value of primary care research should not only be palpable by PCPs, national research funding agencies should also recognise its merits in improving national health beyond economic gain. Funds devoted to PCPs conducting scientifically robust studies will provide an additional impetus for private GPs to engage patients and increase the scale of primary care research. In the long run, an extensive network of PCPs in collaborative research endeavours and serving a wide population with an enlarged pool of potential research subjects will attract research-related investments, which will also, ultimately, benefit the nation's economy. 


\section{Utility and safety evaluation}

Quality improvement (QI) is part and parcel of any advanced family medicine training curriculum, including that of the Graduate Diploma in Family Medicine, the Master in Medicine for Family Medicine and the Family Medicine Residency programmes. ${ }^{(23)}$ The skills acquired through such training can be applied by PCPs in their respective clinical practices. ${ }^{(24)}$ This is thus another avenue by which PCPs can seek the relevant data needed to identify the gaps in patient care or service delivery, prioritise them according to urgency for action, brainstorm for possible solutions to the identified issues, evaluate these interventions for effectiveness, and adopt proven measures in routine practice. PCPs are encouraged to perform the aforementioned on a regular basis, such that each cycle is a step toward achieving their predetermined benchmarks for quality care. Additionally, as manual data retrieval for QI is laborious, PCPs can now invest in increasingly affordable information systems in their practice to generate data for their review and action. Such a worthwhile investment in infrastructure would provide a platform for regular reviews of their care to patients.

Parallel to this QI drive in primary care practices is the implementation of mandatory reporting of adverse events that affect patients. ${ }^{(25)}$ While these incidents are rare, the onus falls on PCPs to conduct periodic patient safety surveillance in their practice and report any ill effects of unorthodox treatment and adulterated medications to the Health Sciences Authority. ${ }^{(25)}$ National and public healthcare institutions also organise regular forums and conferences on quality improvement and patient safety for staff and healthcare professionals. These forums can be configured to become a platform for GPs in private practice to share their experiences and learn from one another. ${ }^{(26)}$ Patient safety is of paramount importance, necessitating a paradigm shift in mindset among PCPs in both the public and private sectors, to build trust and confidence in one another's practice amid a cordial and nonpunitive healthcare environment.

Depending on the availability of a national IT-based clinical data collection system, the local health authority may consider publishing relevant patient quality and safety data to anonymised healthcare providers in order to generate awareness. PCPs can then refer to and compare their data with that of their peers, thus providing a platform for reflection and self-evaluation. ${ }^{(27)}$ Systematic review has shown that publishing data on patient care performance improves quality of care. ${ }^{(28)}$

\section{Practice transformation}

To cater to the rising expectations and complex needs of an educated ageing population, PCPs will need to review their practice set-up, and if necessary, transform it to enhance patients' experience. The public primary care clinics in Singapore have largely adopted the Chronic Care Model in the configuration of their care delivery via system redesign, decision and self-management support, and utility of clinical information systems. ${ }^{(29)}$ Private GPs can adopt and adapt relevant portions of such system-based practices from their public sector counterpart.

An example of system redesign can be seen in the introduction of health monitoring stations (HMS) in polyclinics. ${ }^{(30)}$ Health attendants' roles have changed over time, and they are now trained to monitor patients' clinical parameters such as blood pressure, weight and body mass index at the HMS. Likewise, private GPs can train and empower clinic assistants to perform similar clinical tasks, thus engaging their patients more effectively at their clinics. Private GPs can also tap on the Skills Development Funding to train clinic or healthcare assistants, so as to expand the capabilities of their staff, facilitate patients' interactions and enhance the image of their clinics. ${ }^{(31)}$

Ready access to web-based information has altered patients' behaviour and expectations of consultations with PCPs. Incorporating evidence-based practices is pivotal for the maintenance of the vital doctor-patient relationship in a technologically savvy society. This includes standardised and objective assessment of patients' disease status using validated tools outlined in the local clinical practice guidelines (CPG), with respect to the management of specific diseases. ${ }^{(32)}$ An example is the use of the Asthma Control Test (ACT), which comprises short, multifaceted questionnaires, to determine the clinical status of adult and paediatric patients with asthma. ${ }^{(33)}$ ACT has been introduced in the local CPG on asthma to provide a holistic assessment of patients' asthma control. ${ }^{(34)}$ Asthma assessment using ACT can be performed by private GPs as routine practice. Alternatively, ACT can be performed by patients themselves, or assisted by clinic assistants prior to consultation.

SingHealth Polyclinics has developed 'Asthmacare Buddy', a smartphone application that functions as a decision support tool to empower asthma self-management in patients. ${ }^{(35)}$ Embedded with videos and incorporating links and materials to educate patients on asthma care, the application allows patients to configure a personalised asthma action plan and medications list, according to their doctor's prescription. The application is also intended for free usage by all PCPs, enabling them to track their patients' personal asthma record. This also illustrates how public and private PCPs can share resources, ultimately benefitting their patients. As with any innovation, novel tools and gadgets have to undergo evaluation and research to determine its effectiveness. In light of this, Asthmacare Buddy is currently undergoing evaluation in a research study. This is another area in which public and private PCPs can collaborate to assess new inventions that can potentially transform practices to improve patient care.

Another area that requires the attention of PCPs is the preparedness of their respective practices with regard to the management of epidemics such as influenza and dengue outbreaks. ${ }^{(36)}$ Primary care practices are often the first points of contact when patients present with symptoms at the advent of an epidemic. Polyclinics use their clinical information systems 
to generate data for sentinel surveillance of endemic disease outbreaks. However, this data is bound to be incomplete, as private GPs manage $81 \%$ of all primary care consultations. ${ }^{(2)}$ Thus, the implementation of a similar clinical information system for all PCPs, both public and private, is another possible area of practice transformation. This will augment the data possessed by the Ministry of Health and the Ministry of the Environment and Water Resources, which can then be used to more accurately pinpoint the locality of the outbreaks. Such data will in turn alert private GPs, who can then initiate measures such as the stocking up of relevant personal protection equipment and other precautions to protect patients, clinic staff and themselves. ${ }^{(37)}$

\section{LIMITATIONS}

The six elements assembled in this article on the PAIR UP approach is meant for easy reference and the consideration of PCPs. This approach does not represent an exhaustive or exclusive package; there could be other initiatives beyond the PAIR UP approach that are worthy of implementation for the optimisation of primary care. In this article, the PAIR UP approach is presented as a conceptual framework, which will require multifaceted evaluation to determine its overall effectiveness and whether its outcomes are desirable. However, this is beyond the scope of this article.

\section{CONCLUSION}

The healthcare landscape is now more favourable for the pairing up of both public and private PCPs to deliver quality primary care to the community. The PAIR UP approach requires the polyclinic physicians and private GPs to leverage each other's strengths and mitigate weaknesses to optimise patient care outcomes. However, this approach awaits further evaluation, and if proven effective, it will build trust, cooperation, collegiality and mutual support between the two major stakeholders in Singapore's primary care landscape.

\section{ACKNOWLEDGEMENTS}

The author is grateful to Janet Lau, Elaine Loh, Lucas Goh, Sally Ho, Chow Mun Hong, Sherlin Wong (SingHealth Polyclinics), Tikki Gee (MOH Holdings), Benedict Ho, David Tay (Agency for Integrated Care), Tham Tat Yean, Koh Thuan Wee (Frontier Family Medicine Clinic) and others for their contributions to this article, and their assistance in assembling the resources for this article.

\section{REFERENCES}

1. Gan KY. Speech by Mr Gan Kim Yong, Minister for Health, at the Ministerial Committee on Ageing (MCA) Aged Care Sector Stakeholder's Dialogue on Friday 20 Jan 2012, 9.30AM, Concorde Hotel. In: Ministry of Health [online]. Available at: http://www.moh.gov.sg/content/ moh_web/home/pressRoom/speeches_d/2012/speech_by_mr_ gankimyongministerforhealthattheministerialcommitte.html. Accessed November 2, 2013.

2. Ministry of Health, Singapore. Primary Care Survey 2010 - Profile of
Primary Care Patients. In: Ministry of Health [online]. Available at: http:// www.moh.gov.sg/content/moh_web/home/Publications/information_ papers/2011/primary_care_survey2010profileofprimarycarepatients.html. Accessed November 2, 2013.

3. Central Provident Fund Board. Healthcare Financing Framework In Singapore. In: CPF Board [online]. Available at: http://mycpf.cpf.gov.sg/ CPF/my-cpf/Healthcare/PvdHC2.htm. Accessed November 2, 2013.

4. Ministry of Health, Singapore. Medisave. In: Ministry of Health [online]. Available at: http://www.moh.gov.sg/content/moh_web/home/costs_and_ financing/schemes_subsidies/medisave.html. Accessed November 2, 2013.

5. Agency for Integrated Care. Community Health Assist Scheme. In: CHAS [online]. Available at: http://www.chas.sg/indexpatients.aspx. Accessed November 2, 2013.

6. Agency for Integrated Care. Regional Health Systems (RHS). In: Agency for Integrated Care [online]. Available at: http://www.aic.sg/page.aspx?id=137. Accessed November 2, 2013.

7. Khalik S. More community health centres to support GPs. The Straits Times 2011 Dec 27.

8. Eastern Health Alliance. Community Health Centre. In: Eastern Health Alliance [online]. Available at: http://www.easternhealth.sg/Pages/ programs-chc.aspx. Accessed November 2, 2013.

9. COS Speech by Minister for Health Gan Kim Yong - Better Health for All (Part 1 of 2), 12 March 2013. In: Ministry of Health [online]. Available at: http://www.moh.gov.sg/content/moh_web/home/pressRoom/ speeches_d/2013/COS2013SpeechBetterHealthforAllPart1 of2.html. Accessed November 3, 2013.

10. Singapore Medical Council. Registration for Family Physicians. In: SMC [online]. Available at: http://www.healthprofessionals.gov.sg/content/ hprof/smc/en/leftnav/announcement/registration_forfamilyphysicians. html. Accessed November 3, 2013.

11. Ministry of Health, Singapore. Family Medicine Residency Programme. In: Family Physicians Accreditation Board [online]. Available at: http://www. healthprofessionals.gov.sg/content/hprof/fpab/en/leftnav/becoming_a_ family_physician/family_medicine_residencyprogramme.html. Accessed November 3, 2013.

12. Fellowship Programme. College of Family Physicians Singapore. [online]. Available at: http://cfps.org.sg/programmes/fellowship-programme-fcfps/. Accessed March 11, 2014.

13. Ministry of Health, Singapore. National Electronic Health Record (NEHR). In: Ministry of Health [online]. Available at: http://www.moh.gov.sg/ content/moh_web/home/Publications/educational_resources/2011/ NationalElectronicHealthRecord.html. Accessed November 3, 2013.

14. Ministry of Health Holding. Healthy.SG [online]. Available at: https:// healthy.sg/web/guest/content/about. Accessed March 11, 2014.

15. Beasley JW, Starfield B, van Weel C, Rosser WW, Haq CL. Global health and primary care research. J Am Board Fam Med 2007; 20:518-26.

16. Grimshaw JM, Eccles MP, Lavis JN, Hill SJ, Squires JE. Knowledge translation of research findings. Implement Sci 2012; 7:50.

17. Black N. Why we need observational studies to evaluate the effectiveness of health care. BMJ 1996; 312:1215-8.

18. Giveon S, Kahan E, Kitai E. Factors associated with family physicians' involvement in research in Israel. Acad Med 1997; 72:388-90.

19. Al-Abdullateef $\mathrm{SH}$. A survey of the attitude and practice of research among doctors in Riyadh Military Hospital Primary Care Centers, Saudi Arabia. J Family Community Med 2012; 19:38-42.

20. Curtis P, Dickinson P, Steiner J, Lanphear B, Vu K. Building capacity for research in family medicine: is the blueprint faulty? Fam Med 2003; 35:124-30.

21. Westfall JM, Mold J, Fagnan L. Practice-based research - "Blue Highways" on the NIH Roadmap. JAMA 2007; 297:403-6.

22. Tan NC, Tan YT, Kin PT. Research consultation clinic: impetus towards facilitating primary care research. Asia Pac Fam Med 2013; 12:4.

23. Tudiver F, Click IA, Ward P, Basden JA. Evaluation of a quality improvement curriculum for family medicine residents. Fam Med 2013; 45:19-25.

24. Czabanowska K, Klemenc-Ketis Z, Potter A, et al. Development of a competency framework for quality improvement in family medicine: a qualitative study. J Contin Educ Health Prof 2012; 32:174-80.

25. Ministry of Health, Singapore. Adverse Event Reporting to HSA. In: Ministry of Health [online]. Available at: http://www.moh.gov.sg/content/moh_web/ healthprofessionalsportal/allhealthcareprofessionals/guidelines/adverse_ event_reporting2HSA.html. Accessed November 3, 2013. 
26. Ministry of Health, Singapore. Quality \& Innovation. In: Ministry of Health [online]. Available at: http://www.moh.gov.sg/content/moh_web/home/ our_healthcare_system/qualityinnovation.html. Accessed November 3, 2013.

27. Wise J. Department of Health lists indicators for healthcare professionals to use in quality accounts. BMJ 2009; 338:b2089.

28. Fung CH, Lim YW, Mattke S, Damberg C, Shekelle PG. Systematic review: the evidence that publishing patient care performance data improves quality of care. Ann Intern Med 2008; 148:111-23.

29. Wagner EH, Austin BT, Davis C, et al. Improving chronic illness care: translating evidence into action. Health Aff (Millwood) 2001; 20:64-78.

30. Sing Health Polyclinics. SHP identifies patients' risk of a heart attack with a click of the mouse. In: SingHealth Polyclinics - Newsroom [online]. Available at: http://polyclinic.singhealth.com.sg/AboutSingHealthPolyclinics/ Newsroom/2008/Pages/Home.aspx. Accessed November 3, 2013.

31. Singapore Workforce Development Agency. Workfare Training Support Scheme. In: Employer-based Funding [online]. Available at: http:// www.wda.gov.sg/content/wdawebsite/L102-ForEmployers/L223E007EmployerBasedFund.html. Accessed November 3, 2013.
32. Ministry of Health, Singapore. Clinical Practice Guidelines. In: Ministry of Health [online]. Available at: http://www.moh.gov.sg/content/moh_web/ home/Publications/guidelines/cpg.html. Accessed November 3, 2013.

33. Nathan RA, Sorkness CA, Kosinski M, et al. Development of the asthma control test: a survey for assessing asthma control. J Allergy Clin Immunol 2004; 113:59-65.

34. Ministry of Health, Singapore. Management of Asthma. In: Ministry of Health [online]. Available at: http://www.moh.gov.sg/content/moh_web/ home/Publications/guidelines/cpg/2008/management_of_asthma.html. Accessed November 3, 2013.

35. Sing Health Polyclinics. Patient Care. In: SHP Asthmacare Buddy SingHealth Polyclinics. Available at: http://polyclinic.singhealth.com. sg/PatientCare/PatientResources/Pages/shp-asthmacare-buddy.aspx. Accessed November 3, 2013.

36. Collins N, Litt J, Moore M, Winzenberg T, Shaw K. General practice: professional preparation for a pandemic. Med J Aust 2006; 185(10 Suppl):S66-9.

37. Horvath JS, McKinnon M, Roberts L. The Australian response: pandemic influenza preparedness. Med J Aust 2006; 185(10 Suppl):S35-8. 


\section{SINGAPORE MEDICAL COUNCIL CATEGORY 3B CME PROGRAMME}

\section{(Code SMJ 201403A)}

1. By $2020,15 \%$ of Singapore's population will be 65 years and older.

2. According to the 2010 Primary Care Survey, $14 \%$ of all primary care physicians work in the polyclinics, managing $45 \%$ of all primary care patient attendances for chronic diseases.

3. Singapore's primary healthcare funding involves a complex matrix that factors in health outcomes and the number of residents in any specific community.

4. Only selected patients on the public assistance scheme are allowed to use the funds in their Medisave account to partially offset the bill for selected chronic disease treatment.

5. Medisave deduction is only allowed for outpatient care of selected chronic diseases followed up at polyclinics; it is not applicable for chronic disease treatment at private general practitioner (GP) clinics.

6. Citizens are only allowed to use their own Medisave account for their own chronic disease treatment, so as to ensure that their family members will have enough for their own use in the future.

7. Medisave is contributed solely by employers, and Medifund is a mandatory healthcare savings contributed to by both patients and their employers.

8. Medishield is a catastrophic medical insurance scheme.

9. Asthma and osteoarthritis are examples of selected diseases included under the Chronic Disease Management Programme (CDMP).

10. There is currently an administrative charge of SGD 30 accompanying the use of Medisave funds for outpatient consultations for selected chronic diseases.

11. The Community Health Assist Scheme (CHAS) extends government healthcare cost subsidies to patients visiting participating private GPs and dentists.

12. CHAS covers the cost of medications commonly used to treat selected chronic diseases included under CDMP.

13. Influenza and pneumococcal vaccinations are considered elective treatments for all patients and are not included under CHAS deductible fees.

14. Private GPs included under the CHAS scheme can directly refer patients to subsidised specialist clinics in public hospitals.

15. Children under the age of 16 are not eligible for inclusion under the CHAS scheme.

16. Community health centres, with services such as diabetes-related eye and foot screenings, are set-up to support private GPs in their care of patients.

17. Equipped with facilities and incorporated with a patient information system to integrate with their respective regional healthcare systems (RHS), Family Medicine Clinics are designed to be operated by a group of private GPs.

18. From July 2011 onwards, only physicians listed in the Register of Family Physicians are allowed to register new private clinics.

19. Only physicians working for the public healthcare system can apply for access to their patients' health records via the National Electronic Health Records.

20. $\mathrm{MOH}$ Holdings is developing a National Patient Health Management portal, also known as Healthy.SG.

\section{Doctor's particulars:}

Name in full

MCR number

Specialty:

Email address

\section{SUBMISSION INSTRUCTIONS}

(1) Log on at the SMJ website: http://www.sma.org.sg/publications/smjcurrentissue.aspx and select the appropriate set of questions. (2) Provide your name, email address and MCR number. (3) Select your answers and click "Submit".

\section{RESULTS:}

(1) Answers will be published in the SMJ May 2014 issue. (2) The MCR numbers of successful candidates will be posted online at the SMJ website by 24 April 2014. (3) Passing mark is $60 \%$. No mark will be deducted for incorrect answers. (4) The SMJ editorial office will submit the list of successful candidates to the Singapore Medical Council. (5) One CME point is awarded for successful candidates.

Deadline for submission: (March 2014 SMJ 3B CME programme): 12 noon, 17 April 2014. 